\title{
LUT
}

Lappeenranta

University of Technology

\section{Bacteriorhodopsin Enhances Efficiency of Perovskite Solar Cells}

Das Subhabrata, Wu Congcong, Song Zhaoning, Hou Yuchen, Koch Rainer, Somasundaran Ponisseril, Priya Shashank, Barbiellini Bernardo, Venkatesan

Renugopalakrishnan

This is a Post-print

published by American Chemical Society

in ACS Applied Materials and Interfaces version of a publication

DOI: 10.1021/acsami.9b06372

Copyright of the original publication: (c) 2019 American Chemical Society

Please cite the publication as follows:

Das S., Wu C., Song Z., Hou Y., Koch R., Somasundaran P., Priya S., Barbiellini B., Venkatesan R. (2019). Bacteriorhodopsin Enhances Efficiency of Perovskite Solar Cells. ACS Applied Materials and Interfaces, Vol 11, Issue 34. p. 30728-30734. DOI: 10.1021/acsami.9b06372 


\section{Bacteriorhodopsin enhances Efficiency of Perovskite solar cells}

Subhabrata Das, ${ }^{\ddagger}$ Congcong Wu, ${ }^{\llbracket}$ Zhaoning Song, ${ }^{\S}$ Yuchen Hou, ${ }^{\natural}$ Rainer Koch,"

Ponniseril Somasundaran, ${ }^{\ddagger}$ Shashank Priya, ${ }^{, \pi}$ Bernardo Barbiellini, ${ }^{, * \perp}, \#$ and

Renugopalakrishnan Venkatesan ${ }^{*},{ }^{\circ} \mathrm{O}$

${ }^{\ddagger}$ Langmuir Center of Colloids and Interfaces, Columbia University in the City of New York, New York, NY 10027, USA

"Materials Science and Engineering, Penn State, University Park, PA 16802, USA

${ }^{\S}$ Department of Physics, University of Toledo, OH 43606, United States

"Institute of Chemistry, Carl von Ossietzky University Oldenburg, 114-118, 26129,

Oldenburg, Germany

${ }^{\perp}$ Department of Physics, LUT University, FI-53851 Lappeenranta, Finland

\#Department of Physics, Northeastern University, Boston, Massachusetts 02115, USA

${ }^{\circledR}$ Department of Chemical Biology, Northeastern University, Boston, Massachusetts 02115, United States

${ }^{\circ}$ Department of Biophysics, Children's Hospital, Harvard Medical School, Boston, Massachusetts 02115, USA

E-mail: sup103@psu.edu; bernardo.barbiellini@lut.fi;

v.renugopalakrishnan@northeastern.edu

IIn honor of Prof. Seeram Ramakrishna, National University of Singapore 


\begin{abstract}
In recent years, halide perovskites have upstaged decades of development in solar cells by reaching power conversion efficiencies that surpasses polycrystalline silicon performance. The efficiency improvement in the perovskite cells is related to repeated recycling between photons and electron-hole pairs, reduced recombination losses and increased carrier lifetimes. Here, we demonstrate a novel approach towards enhancing the efficiency of perovskite solar cells by invoking the Forster Resonance Energy Transfer (FRET) mechanism. FRET occurs in the near-field region as bacteriorhodopsin (bR) protein and perovskite have similar optical gaps. Titanium dioxide functionalized with bR protein is shown to accelerate the electron injection from excitons produced in the perovskite layer. FRET predicts the strength and range of exciton transport between separated perovskite and bR layers. We show that the cells incorporating $\mathrm{bR} / \mathrm{TiO}_{2}$ layers exhibit much higher photovoltaic performance. These results open the opportunity to develop a new class of bio-perovskite solar cells with improved performance and stability.
\end{abstract}

\title{
Keywords
}

FRET; Purcell Effect; Photoluminescence; Fill Factor; Perovskite; Bacteriorhodopsin 


\section{INTRODUCTION}

Organic and inorganic hybrid perovskites represent a new class of semiconductors with promising characteristics for a wide range of optoelectronic applications. Hybrid perovskites display many advantages such as long electron-hole diffusion lengths, simple fabrication procedure, sustainable chemistry, and cost-effective raw materials. Perovskites are represented by the generic formula, $\mathrm{ABX}_{3}$, in a close-packed quasi-cubic unit cell. The A-site cations, e.g. methylammonium $\left(\mathrm{CH}_{3} \mathrm{NH}_{3}{ }^{+}\right)(\mathrm{MA})$, formamidinium $\left(\mathrm{HC}\left(\mathrm{NH}_{2}\right)_{2}{ }^{+}\right)(\mathrm{FA})$ or cesium $\left(\mathrm{Cs}^{+}\right)$), occupy the corners of the cube; the B-site cations are typically dominated by group IVA metals with divalent oxidation state $\left(\mathrm{Pb}^{2+}, \mathrm{Sn}^{2+}, \mathrm{Ge}^{2+}\right)$; the anion $\mathrm{X}$ is generally a halide $\left(\mathrm{Cl}^{-}, \mathrm{Br}^{-}\right.$, or $\left.\mathrm{I}^{-}\right)$. In recent years, hybrid perovskite based solar cells with power conversion efficiencies up to $\geq 23 \%^{1,2}$ have been demonstrated due to advancements in solution-synthesis and film-deposition techniques. Such an improvement has been the result of the development of new fabrication methods and the design of materials layers with proper band alignment that promotes charge transport.

In case of photovoltaic power conversion efficiency, there are several factors that contribute towards the improved performance: (1) composition-driven tunable bandgap spanning a wide range of the solar spectrum ${ }^{3}$; (2) direct band-gap with high absorption coefficients (high density of states above and below the band gap near the quasi-Fermi level); ${ }^{4}$ (3) low effective masses and relatively high carrier mobilities; (4) long carrier diffusion lengths; (5) low excitonic binding energy values; (6) high photoluminescence quantum yield ${ }^{5}$ and (7) highly efficient radiative recombination rates due to sharp band edges and absence of mid-gap states. The variation of the bandgap as a function of composition and doping level has been widely studied in hybrid perovskites. By varying cations on $\mathrm{A}$ and $\mathrm{B}$ site or anion $\mathrm{X}$, a wide range of bandgaps can be achieved ranging from 1.2-2.3 eV. In typical perovskite solar cells (PSCs), several-hundred nanometer thick light absorbing layer, with or without a mesoporous scaffold, is sandwiched between the electron and hole transport layers (ETLs and HTLs, respectively). Upon the absorption of incident photons, carriers are created in the absorber that travel through a transport pathway including the ETL or HTL to the electrodes. The low non-radiative recombination rates and high photoluminescence (PL) yields of these materials allow one photo-excited state 
to undergo multiple radiative emission-absorption events before it is lost through nonradiative decay. Repeated recycling between photons and electron-hole pairs creates charge extraction over large length scales, and high excitation densities within the perovskite layer allow high open-circuit voltages. ${ }^{6}$ The architecture of PSCs is originated from the dyesensitized solar cell (DSSC), where mesoporous $\mathrm{TiO}_{2}$ layer is employed as a scaffold layer. ${ }^{7}$ The high surface area of the $\mathrm{TiO}_{2}$ mesoporous layer allows the sufficient adsorption of light absorbing molecules for energy conversion. Thavasi et al. demonstrated the feasibility of bacteriorhodopsin (bR) as bio-photosensitizer for $\mathrm{TiO}_{2}$ in the so-called biosensitized solar cell (BSSC). ${ }^{8}$ In the BSSC, bR works as a light-driven proton pump during charge separation initiated by photon absorption, with simultaneous electron ejection in the $\mathrm{TiO}_{2}$ layer. The sensitization of $\mathrm{TiO}_{2}$ with bR has been further rationalized by firstprinciples calculations performed by Koch et al. ${ }^{9}$ and has been used by Allam et al. ${ }^{10}$ for enhancing the photoelectrochemical water splitting.

Here we developed a new type of bio-perovskite solar cell (BPSC), as illustrated in Figure 1. The band alignment is illustrated in Figure 1a and the schematic architecture of the BPSC is displayed in Figure 1b. The valence band maximum (VBM) and conduction band maximum $(\mathrm{CBM})$ for the $\mathrm{CH}_{3} \mathrm{NH}_{3} \mathrm{PbI}_{3}\left(\mathrm{MAPbI}_{3}\right)$ are reported to be 5.3 and $3.7 \mathrm{eV}$, respectively, ${ }^{11}$ and the highest occupied molecular orbital (HOMO) of spiro-OMeTAD is located at $5.2 \mathrm{eV} .{ }^{12}$ The favorable band alignment of perovskite with $\mathrm{TiO}_{2}$ ETL and SpiroOMeTAD HTL allows the charge carrier extraction to electrodes. The key component of the BPSC is the bR molecules between $\mathrm{TiO}_{2}$ and perovskite layers, which implements the energy-transfer step involving photon absorption and hot carriers transfer. The transfer of light energy absorbed by perovskite to bR occurs first via an efficient Forster resonance energy transfer (FRET) ${ }^{13,14}$ and further, from bR quantum dots (QD) to $\mathrm{TiO}_{2}$, via the electron ejection mechanism of the $\mathrm{bR}$ proton pump mentioned above. The enhanced thermal stability of $\mathrm{bR}$ and its mutants, accomplished by rational site-directed mutations, provides additional advantages. The operation of these three transformative BPSC paradigms holds great promise for construction of robust and efficient electrolyte free solid-state photovoltaic devices.

\section{EXPERIMENTAL SECTION}




\subsection{Synthesis of $\mathrm{TiO}_{2} / \mathrm{bR}$ Layer}

Cys bR mutant was immobilized on $\mathrm{TiO}_{2}$ substrate by covalent attachment of Ti to Cys groups using standard experimental protocol. ${ }^{8}$ The details of the synthesis and purification of bR mutants were provided in Supporting Information. bR concentration of $6.67 \mathrm{~g} / \mathrm{L}$ was diluted 10 times in suspension buffer $(150 \mathrm{mM} \mathrm{KCl}, 10 \mathrm{mM}$ Tris-HCl, $\mathrm{pH} 8.2)$ to achieve a final absorbance of 0.2 to 0.3 at $570 \mathrm{~nm}$. We mixed $0.5 \mathrm{~mL}$ of this diluted bR solution in suspension buffer with $250 \mu \mathrm{L}$ of incubation buffer $(300 \mathrm{mM} \mathrm{KCl}, 10 \mathrm{mM}$ Tris- $\mathrm{HCl}, \mathrm{pH}$ 7.82), and this mixture was placed on the glass substrate coated with $\mathrm{TiO}_{2}$ incubated for 6 hr. The container was kept in the dark at room temperature during the attachment process. After $6 \mathrm{hr}$, the substrate was rinsed with incubation buffer several times, followed by drying at room temperature in the dark for 5 to $6 \mathrm{hr}$. Care was taken to keep the substrate always under dark conditions. After rinsing, visual inspection did show pink and purple spots on the $\mathrm{TiO}_{2}$ substrates.

\subsection{Perovskite Solution Preparation}

The perovskite thin film was synthesized by exfoliating perovskite crystals and selfassembling to thin film. ${ }^{15}$ To prepare the $\mathrm{MAPbI}_{3}$ crystals, $1.2 \mathrm{M} \mathrm{MAPbI}_{3}$ solution was fabricated by dissolving $\mathrm{PbI}_{2}$ and $\mathrm{MAI}$ (molar ratio 1:1) in $\gamma$-butyrolactone and filtered in the vial. Next, the vial with $\mathrm{MAPbI}_{3}$ solution was placed in an oil bath and slowly heated to $120{ }^{\circ} \mathrm{C}$ and kept for $3 \mathrm{~h}$ for $\mathrm{MAPbI}_{3}$ crystal growth. The crystals were washed with diethyl ether and dried under nitrogen gas. The $\mathrm{MAPbI}_{3}$ crystals were exfoliated by exposing to methylamine $\mathrm{CH}_{3} \mathrm{NH}_{2}$ gas. The $\mathrm{MAPbI}_{3}$ crystals were placed in a vial and then packed in a sealed bottle with methylamine ethanol solution for $12 \mathrm{~h}$. The evaporated methylamine gas diffused into the vial and reacted with $\mathrm{MAPbI}_{3}$ crystals, forming a liquid perovskite intermediate. The liquid perovskite was dissolved in acetonitrile to form $1 \mathrm{M}$ perovskite solution.

\subsection{Device Fabrication and Characterization}

The $\mathrm{TiO}_{2}$ compact layer was fabricated by spin-coating titanium isopropoxide (TTIP) ethanol solution on FTO glass at $2000 \mathrm{rpm}$ for $20 \mathrm{~s}$, followed by annealing at $500{ }^{\circ} \mathrm{C}$ for 1 h. To fabricate the $\mathrm{TiO}_{2}$ mesoporous layer, the $\mathrm{TiO}_{2}$ solution prepared by diluting $18 \mathrm{NR}-\mathrm{T}$ 
paste with $\alpha$-terpineol and ethanol was spin-coated on the top of $\mathrm{TiO}_{2}$ compact layer. $\mathrm{TiO}_{2}$ layer was annealed at $500{ }^{\circ} \mathrm{C}$ for $1 \mathrm{~h}$. The perovskite thin film was deposited by spin coating the perovskite solution onto the $\mathrm{TiO}_{2}$ layer at $4000 \mathrm{rpm}$ for $20 \mathrm{~s}$ and then heated at $100{ }^{\circ} \mathrm{C}$ for $10 \mathrm{~min}$. The Spiro-OMeTAD solution was spin-coated on the perovskite layer at 4000 rpm for $20 \mathrm{~s}$ serving as the hole transport layer. Lastly, $80 \mathrm{~nm}$ of gold was thermally evaporated as the metal electrode, keeping the active area of each device at $0.096 \mathrm{~cm}^{2}$. The steady-state and transient photoluminescence spectra were measured by FLS1000 Photoluminescence Spectrometer (Edinburgh Instruments Ltd). The samples were excited by a pulsed laser with a wavelength of $510 \mathrm{~nm}$ and illuminated from the film side for excitation. The Oriel Sol 3A Class AAA Solar Simulator (Newport) was employed to provide air mass (AM 1.5) illumination of $100 \mathrm{~mW} \mathrm{~cm}^{-2}$. The samples were illuminated from the left (the front side) by the monochromatic (continuous) flux of photons. However, the model can be extended to an arbitrarily positioned (monochromatic or spectral) illumination source due to photon recycling and recombination events. The J-V curve was measured by Keithley digital source meter (Model 2440). The incident photon-to-current conversion efficiency (IPCE) spectra were measured by QUANTX-300 system (Newport) and the Electrochemical Impedance spectroscopy (EIS) was performed by using $600 \mathrm{E}$ electrochemical station ( $\mathrm{CH}$ Instruments, Inc.).

\section{RESULTS AND DISCUSSION}

\section{1 bR Assisted Charge Transfer}

The bR molecules are integrated within perovskite solar cells by attaching on the surface of $\mathrm{TiO}_{2}$ nanoparticles of the mesoporous layer to enhance the charge transfer between perovskite and $\mathrm{TiO}_{2}$ ETL. Figure 2a shows the cross-sectional scanning electron microscopy (SEM) image of the BPSC, where the compact $\mathrm{TiO}_{2}$ layer, perovskite/mesoporous $\mathrm{TiO}_{2} / \mathrm{bR}$, Spiro-OMeTAD, and Au electrode were deposited on the FTO glass, respectively. The thickness of perovskite and mesoporous $\mathrm{TiO}_{2} / \mathrm{bR}$ is approximately $500 \mathrm{~nm}$, which can sufficiently absorb the light. The top-view SEM of the perovskite layer deposited on $\mathrm{TiO}_{2} / \mathrm{bR}$ was presented in Figure S1, showing a uniform, pinhole-free perovskite film with a grain size of around $200 \mathrm{~nm}$. Upon light excitation, the photogenerated electrons from both capping perovskite layer and penetrated perovskite 
will be transferred into the mesoporous $\mathrm{TiO}_{2}$ by the assistance of the bR molecules. To study the coupling between $\mathrm{bR}$ and $\mathrm{TiO}_{2}$, we measured the photoluminescence (PL) spectra for pristine $\mathrm{TiO}_{2}$ film and $\mathrm{TiO}_{2} / \mathrm{bR}$ film. The $\mathrm{TiO}_{2}$ mesoporous film was immersed in bR solution for adsorption of bR on the surface of $\mathrm{TiO}_{2}$ nanoparticles. As shown in Figure $2 \mathrm{~b}$, the pristine $\mathrm{TiO}_{2}$ film showed no emission in the wavelength range from $600 \mathrm{~nm}$ to 850 $\mathrm{nm}$. Upon the adsorption of bR, there is a clear emission peak around $720 \mathrm{~nm}$, which can be attributed to the radiative recombination of electrons and holes excited from the bR molecules, indicating that $\mathrm{bR}$ is bonding on the $\mathrm{TiO}_{2}$ surface. The absorption spectrum of the $\mathrm{bR} / \mathrm{TiO}_{2}$ was presented in Figure $\mathrm{S} 2$, showing a distinctive absorption feature of $\mathrm{bR}$ molecules. We then deposited $\mathrm{MAPbI}_{3}$ perovskite on top of $\mathrm{TiO}_{2}$ film and $\mathrm{TiO}_{2} / \mathrm{bR}$ film, respectively, and measured the PL spectra, as shown in Figure 2c. The emission peak for $\mathrm{MAPbI}_{3}$ is at $768 \mathrm{~nm}$, corresponding to a bandgap of $\sim 1.6 \mathrm{eV}$. Interestingly, with the adsorption of bR on $\mathrm{TiO}_{2}$ film, the intensity of the perovskite PL decreased significantly. The effect implies that there is a charge carrier extraction at the interface between the perovskite and $\mathrm{bR}$, decreasing the radiative recombination inside the perovskite and thus reducing the PL intensity. In addition, the emission peak is blue shifted from $768 \mathrm{~nm}$ to $762 \mathrm{~nm}$, which can be attributed to the FRET with the exciton transfer from perovskite to the bR molecules. To understand the energy and charge transfer kinetics, we measured the time-resolved PL spectra to extract the charge carrier lifetime, as shown in Figure 2d. The stretched exponential PL decay can be expressed as:

$I(t)=\sum_{i=1}^{n} I_{i} e^{-\left(\frac{t}{\tau i}\right)^{\beta i}}$

where $I(t)$ is the fluorescence intensity at time $t, n$ is the number of components, $I_{i}$ is the initial intensity of each component, $\tau_{i}$ is the characteristic lifetime and $\beta_{i}$ is the stretching exponent $\left(0<\beta_{i} \leq 1\right)$. $\beta$ deforms the exponential so that the initial decay is faster and the tail longer. The lifetime of a stretched exponential decay is quantified by its mean relaxation time $\langle\tau\rangle$ :

$\tau=\frac{\tau_{c}}{\beta} \int_{0}^{\infty} x^{(1-\beta) / \beta} e^{-x} d x$ 
The lifetime calculated from the PL decay is $\tau_{1}=1.31 \mathrm{~ns}, \tau_{2}=9.55 \mathrm{~ns}, \tau_{3}=24.47 \mathrm{~ns}$ for $\mathrm{TiO}_{2} /$ perovskite. In the case of $\mathrm{TiO}_{2} / \mathrm{bR} /$ perovskite, the lifetime analysis yields: $\tau_{1}=0.89$ $\mathrm{ns}, \tau_{2}=3.63 \mathrm{~ns}, \tau_{3}=12.17 \mathrm{~ns}$. Clearly, the lifetime of the excited electron is decreased significantly upon the adsorption of $\mathrm{bR}$ on $\mathrm{TiO}_{2}$ film, which further confirms the bR can assist the charge carrier extraction from perovskite to $\mathrm{TiO}_{2} \mathrm{ETL}$.

To study the effect $b R$ molecules on the conversion efficiency of perovskite solar cells, the J-V characteristics of the two devices were measured, as shown in Figure 3a. The conventional cell $\left(\mathrm{TiO}_{2}\right.$-PSC) showed $J_{s c}$ of $22.59 \mathrm{~mA} \mathrm{~cm}{ }^{-2}, V_{o c}$ of $1.02 \mathrm{~V}$, FF of 0.618 , corresponding to the efficiency of $14.59 \%$. The perovskite solar cell with $\mathrm{bR}\left(\mathrm{TiO}_{2} / \mathrm{bR}\right.$ PSC) exhibited $J_{s c}$ of $22.61 \mathrm{~mA} \mathrm{~cm}{ }^{-2}, V_{o c}$ of $1.05 \mathrm{~V}, \mathrm{FF}$ of 0.705 , with the conversion efficiency of $17.02 \%$. The histogram of the conversion efficiency (Figure S3) shows majority devices present efficiency over $16 \%$, and the average efficiency is $16.34 \%$. The efficiency improvement with the bR is mainly attributed to the increased fill factor, from 0.618 to 0.705 , which is due to the fast electron transfer from perovskite to $\mathrm{bR}$ and then $\mathrm{TiO}_{2}$, reducing the electron transport resistance at the perovskite/ $/ \mathrm{TiO}_{2}$ interface. Figure $3 \mathrm{~b}$ shows the incident-photon-to-charge conversion efficiency (IPCE) spectrum of the BPSC. The maximum external quantum efficiency (EQE) is over $95 \%$ at the wavelength of 531 $\mathrm{nm}$ and the EQE response edge is at around $770 \mathrm{~nm}$, which is consistent with the absorption edge of $\mathrm{MAPbI}_{3}$ perovskite. The integrated $\mathrm{J}_{s c}$ calculated from the IPCE spectrum is 22.5 $\mathrm{mA} \mathrm{cm}{ }^{-2}$, which is consistent with the $\mathrm{J}_{s c}$ from J-V curve. The forward and reverse scan J$\mathrm{V}$ characteristics were performed for both PSCs, as shown in Figure S4. The $\mathrm{TiO}_{2}-\mathrm{PSC}$ exhibited a reverse-scan efficiency of $14.59 \%$ and a lower efficiency of $12.66 \%$ with forward scan. In contrast, the $\mathrm{TiO}_{2} /$ bR-PSC exhibited a reverse-scan efficiency of $17.02 \%$ and a forward-scan efficiency of $16.26 \%$, showing a much less hysteresis, which can be ascribed to the enhanced charge transfer assisted by the bR molecules, releasing the interfacial charges at the $\mathrm{ETL} /$ perovskite interface. Electrochemical impedance spectroscopy (EIS) was measured to study the interfacial charge carrier transfer behavior. Figure $3 \mathrm{c}$ and $3 \mathrm{~d}$ compare the Nyquist plots for the PSCs with and without bR in dark and illuminated conditions, respectively. The charge transfer resistance of the $\mathrm{TiO}_{2}-\mathrm{PSC}$ and $\mathrm{TiO}_{2} / \mathrm{bR}-\mathrm{PSC}$ is $309.2 \Omega$ and $177.5 \Omega$ in the dark; $30.6 \Omega$ and19.2 $\Omega$ in the 1 sun illumination condition, respectively. This reduction in the transfer resistance is attributed 
to the enhanced charge extraction assisted by the bR molecules, rationalizing the increase of FF for the BPSC.

\subsection{Theoretical Interpretation}

The present results can be rationalized in terms of a FRET model. The lowest unoccupied molecular orbital (LUMO) and highest occupied molecular orbital (HOMO) of bR is determined by surface photo-voltage spectroscopy (SPS) to be $3.8 \mathrm{eV}$ and $5.4 \mathrm{eV}$ following Li et al. ${ }^{16}$ The details of the Density Functional Theory (DFT) calculation for molecular orbitals of retinal in $\mathrm{bR}$ is given in the Supporting Information and the electronic transitions between the HOMO and LUMO in the retinal was illustrated in Figure S5. Since both $\mathrm{MAPbI}_{3}$ and $\mathrm{bR}$ have almost the same gap they are resonantly coupled and FRET occurs in the near-field region. The efficiency and the spatial range of this mechanism for exciton transfer can be estimated using the near-field resonance of electric dipoles as shown by King et al. ${ }^{14}$ The shortening of the perovskite emission lifetime upon the adsorption of bR on $\mathrm{TiO}_{2}$ film is a clear proof that FRET provides an effective way to achieve the Purcell effect in our solar cell. ${ }^{17,18}$ From the lifetime change, one can estimate the FRET rate as about $0.36(1 / \mathrm{ns})$. As mentioned earlier, the main effect of $\mathrm{bR}$ is manifested in increase in the FF of BPSC. FF is a parameter which quantifies the rectification of a solar cell device within the fourth quadrant of the $\mathrm{J}-\mathrm{V}$ characteristics where power is generated. FF is influenced by charge carrier recombination losses which are significantly reduced by FRET.

In the present model shown in Figure $4 \mathrm{a}, \mathrm{MAPbI}_{3}$ replaces the semiconductor quantum dot originally considered by King et al. ${ }^{14}$ The FRET coupling strength $U$ is determined by the relation:

$U=\frac{\kappa D_{P e} D_{b R}}{S_{r} R^{3}}$

where $s_{r}$ is the permittivity of the medium, $R$ is the separation between the molecules, $D_{P e}$ and $D_{b R}$ are the dipole moments of the Perovskite and $\mathrm{bR}$, respectively, and the angular factor $\kappa$ depends on the orientations of dipoles relative to the separation between molecules.

We use the dipole values of $D_{P} e=1.94$ and $D_{b R}=40$ from literature. ${ }^{19,20}$ We assume the medium dry and take a permittivity $s_{r}=1, \kappa=1$ and keep the bR-perovsite separation 
distance $R$ as a free parameter. The model also needs another parameter $\gamma$ which is the inverse lifetime of an exciton in $\mathrm{bR}$, and can be determined to be around $0.05 \mathrm{eV}$ according to King et al. ${ }^{14}$ Other rates can be neglected in the calculations since they are much smaller than $\gamma$. Figure $4 \mathrm{~b}$ shows the FRET efficiency as a function of $R$. For separations $R$ larger than the FRET radius $R_{0}$ the efficiency is less than $50 \%$. Thus, in the present model, FRET radius $R_{0}$ ranges between $4-7 \mathrm{~nm}$ if $\gamma$ varies between 0.05 and $0.1 \mathrm{eV}$. Figure $4 \mathrm{c}$ illustrates that for $\mathrm{R}=2 \mathrm{~nm}$, the exciton transfer from perovskite to $\mathrm{bR}$ occurs within a few hundred femtoseconds. Interestingly, the exciton transfer presents a damped oscillatory behavior shown by the blue curve in Figure $4 \mathrm{c}$ when $\gamma=0.05$. This feature is not captured by previous FRET models and reveals that exciton transport can be coherent-like rather than diffusionlike.

\section{CONCLUSION}

We have developed a BPSC that utilizes novel optoelectronic characteristics of perovskite and $b R$. The concept of photon recycling utilized to explain the perovskite success is combined here with FRET to achieve significant improvement in the performance of PSCs. FRET is possible because of $\mathrm{bR}$ and perovskite possessing similar optical gaps. The perovskite acts as an antenna for photon absorption, with subsequent transfer to the retinal complex in bR. The retinal complex in bR is known to be an efficient absorber of photons through direct capture, and a similar efficiency is found for non-radiative transfer of excitons from perovskite to $\mathrm{bR}$ via the FRET mechanism. The decrease in photoluminescence by photosensitive bR indicates that FRET plays an important role in the excitonic transport. Thus, the present increase of FF can be explained by the fact that charge carrier recombination losses are significantly reduced by FRET process. In the future research, the nanostructure and the chemical bonding between $\mathrm{bR}$ and $\mathrm{TiO}_{2}$ can be modified for enhancing the proton pumping property of $b R$ and facilitating charge transfer, which is expected to further improve the efficiency of BPSC. This work opens a new direction to utilize the unique functionalities of biomaterials in photovoltaics for improving the charge transfer and thus the photo conversion performance. 


\section{ASSOCIATED CONTENT}

\section{Supporting Information}

The procedure of synthesizing bR mutants; Top-view SEM of the perovskite layer deposited on $\mathrm{TiO}_{2} / \mathrm{bR}$ mesoporous layer; The UV-vis absorption spectrum of $\mathrm{bR} / \mathrm{FTO}$ glass by spin coating and dip coating, and the $\mathrm{TiO}_{2} / \mathrm{bR}$ sample; Statistical power conversion efficiency (PCE) distribution of bR-incorporated PSC; Forward and reverse scan J-V characteristics of $\mathrm{TiO}_{2}$-PSC and $\mathrm{TiO}_{2} /$ bR-PSC; Density Functional Theory (DFT) calculation for molecular orbitals of retinal in $\mathrm{bR}$.

\section{Acknowledgments}

C.W. and S.P. acknowledge the support from Air Force Office of Scientific Research (AFOSR) through grant number FA9550-18-1-0233 (Biophysics and Natural Materials). We thank Kai Zhu from NREL for taking part in the fruitful discussions in the proposal of Perovskite architecture. VR thanks Wallace Coulter Foundation, Rothschild Foundation

for support, Prof. Esteve Padros, Universitaria Autonoma de Barcelona, Espagna, Professor Norbert Hampp from Philipps-Universitat Marburg, Allemagne and late Prof. Har Gobind Khorana, MIT, Cambridge, MA, USA for help with Cys bR mutant.

\section{AUTHOR INFORMATION}

E-mail: sup103@psu.edu;

E-mail: bernardo.barbiellini@lut.fi;

E-mail: v.renugopalakrishnan@,northeastern.edu

\section{Notes}

The authors declare no competing financial interest.

\section{REFERENCES}

(1) Etgar, L.; Gao, P.; Xue, Z.; Peng, Q.; Chandiran, A. K.; Liu, B.; Nazeeruddin, M. K.; Grätzel, M. Mesoscopic CH3NH3PbI3/TiO2 Heterojunction Solar Cells. Journal of the American Chemical Society 2012, 134, 17396-17399, PMID: 23043296.

(2) Zhao, Y.; Zhu, K. Organic-inorganic Hybrid Lead Halide Perovskites for Optoelectronic and Electronic Applications. Chemical Society Reviews 2016, 45, 655-689. 
(3) Sadhanala, A.; Ahmad, S.; Zhao, K. C.; Bein, T.; Docampo, P. Blue-green color Tunable Solution Processable Organolead Chloride-Bromide Mixed Halide Perovskites for Optoelectronic Applications. Nano letters 2015, 15, 6095-6101.

(4) Lee, M. M.; Teuscher, J.; Miyasaka, T.; Murakami, T. N.; Snaith, H. J. Efficient Hybrid Solar Cells based on Meso-Superstructured Organometal Halide Perovskites. Science 2012, 338, 643-647.

(5) Tan, Z.-K.; Moghaddam, R. S.; Lai, A.; Pazos, L. M.; Credgington, D. Bright LightEmitting Diodes based on Organometal Halide Perovskite. Nature nanotechnology 2014, 9, 687 .

(6) Pazos-Outón, L. M.; Szumilo, M.; Lamboll, R.; Richter, J. M.; Crespo-Quesada, M.; Abdi-Jalebi, M.; Beeson, H. J.; Vrućinić, Photon Recycling in Lead Iodide Perovskite Solar Cells. Science 2016, 351, 1430-1433.

(7) O'regan, B.; Grätzel, M. A low-cost, High-Efficiency Solar Cell based on Dye-Sensitized Colloidal TiO2 films. nature 1991, 353, 737.

(8) Thavasi, V.; Renugopalakrishnan, V.; Jose, R.; Ramakrishna, S. Controlled Electron Injection and Transport at materials interfaces in Dye Sensitized Solar Cells. Materials Science and Engineering: R: Reports 2009, 63, 81-99.

(9) Koch, R.; Lipton, A. S.; Filipek, S.; Renugopalakrishnan, V. Arginine interactions with Anatase TiO2 (100) Surface and the perturbation of 49Ti NMR chemical shifts - a DFT investigation: relevance to Renu-Seeram bio solar cell. Journal of Molecular Modeling 2011, 17, 1467-1472.

(10)Allam, N. K.; Yen, C.-W.; Near, R. D.; El-Sayed, M. A. Bacteriorhodopsin/TiO 2 Nanotube arrays Hybrid system for Enhanced Photoelectrochemical Water splitting. Energy \& Environmental Science 2011, 4, 2909-2914.

(11)Docampo, P.; Ball, J. M.; Darwich, M.; Eperon, G. E.; Snaith, H. J. Efficient Organometal trihalide Perovskite Planar-Heterojunction solar cells on flexible Polymer Substrates. Nature communications 2013, 4, 2761.

(12)Kim, H.-S.; Lee, C.-R.; Im, R.; Yum, J.-H.; Moser, J. E. Lead Iodide Perovskite Sensitized all-solid-state submicron thin film Mesoscopic solar cell with Efficiency exceeding 9\%. Scientific reports 2012, 2, 591.

(13)Renugopalakrishnan, V.; Barbiellini, B.; King, C.; Molinari, M.; Mochalov, K.; Sukhanova, A.; Nabiev, I.; Fojan, P.; Tuller, H. L.; Chin, M.; Somasundaran, P.; Padrós, E.; Ramakrishna, S. Engineering a Robust Photovoltaic Device with Quan- tum Dots and Bacteriorhodopsin. The Journal of Physical Chemistry C 2014, 118, 16710-16717, PMID: 25383133.

(14)King, C.; Barbiellini, B.; Moser, D.; Renugopalakrishnan, V. Exactly Soluble model of Resonant Energy Transfer between molecules. Physical Review B 2012, 85, 125106. 
(15)Wu, C.; Li, H.; Yan, Y.; Chi, B.; Pu, J.; Li, J.; Sanghadasa, M.; Priya, S. Cost- effective sustainable-engineering of $\mathrm{CH} 3 \mathrm{NH} 3 \mathrm{PbI} 3$ Perovskite Solar Cells through slicing and restacking of 2D layers. Nano Energy 2017, 36, 295-302.

(16)Li, L. S.; Xu, T.; Zhang, Y. J.; Jin, J.; Li, T. J.; Zou, B.; Wang, J.-P. Photovoltaic characteristics of BR/p-silicon Heterostructures using Surface Photovoltage Spectroscopy. Journal of Vacuum Science \& Technology A: Vacuum, Surfaces, and Films 2001, 19, 1037-1041.

(17)Pelton, M. Modified Spontaneous Emission in Nanophotonic Structures. Nature Photonics 2015, 9, 427.

(18)Barbiellini, B.; Das, S.; Renugopalakrishnan, V.; Somasundaran, P. Electromagnetic Field in Hybrid Quantum Plasmonic-Photonic Systems. Condensed Matter 2018, 3, 10.

(19)Senanayak, S. P.; Yang, B.; Thomas, B.; Goedel, K.; Guha, S.; Moya, X. Understanding Charge Transport in Lead Iodide Perovskite thin-film Field-effect Transistorsransistors. Science advances 2017, 3, e1601935.

(20)Kietis, B.; Macernis, M.; Sulskus, J.; Valkunas, L. Estimation of the Permanent Dipole moment of Bacteriorhodopsin. Lithuanian Journal of Physics 2010, 50 . 
(a)

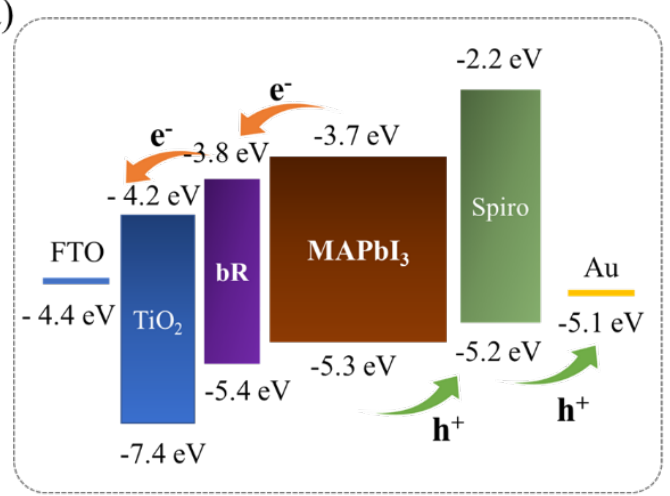

(b)

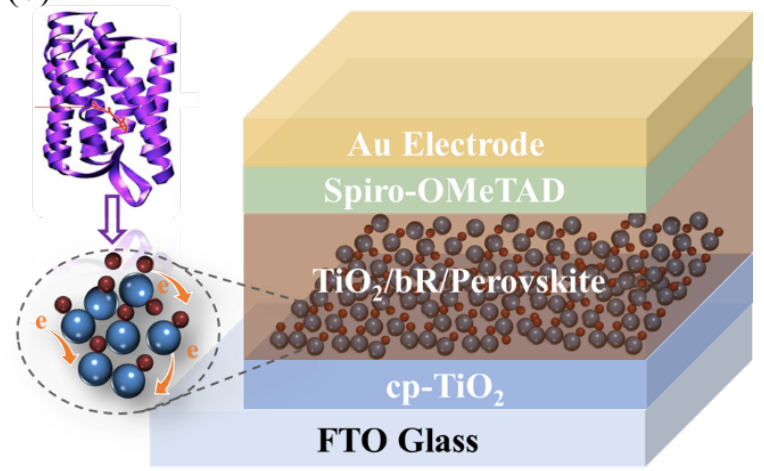

Figure 1. (a) Band alignment of the bR/perovskite solar cell (BPSC). (b) Schematic of the $\mathrm{BPSC}$ architecture, where the compact $\mathrm{TiO}_{2}$ layer, perovskite/mesoporous $\mathrm{TiO}_{2} / \mathrm{bR}$, SpiroOMeTAD, and Au electrode were deposited on the FTO glass, respectively. 
(a)

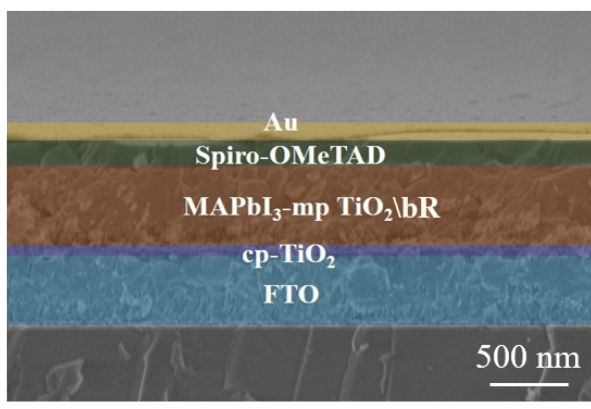

(c)

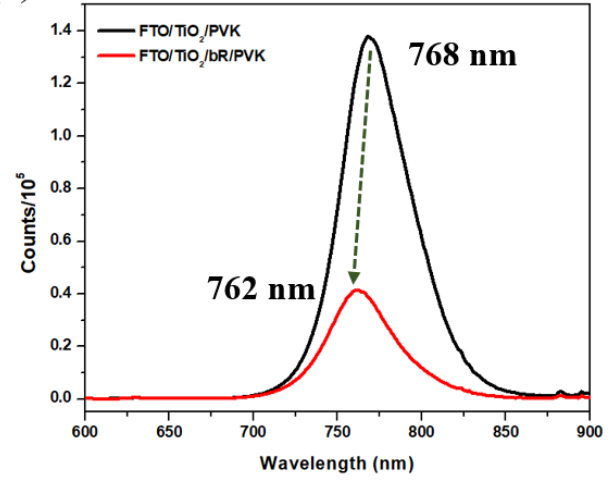

(b)

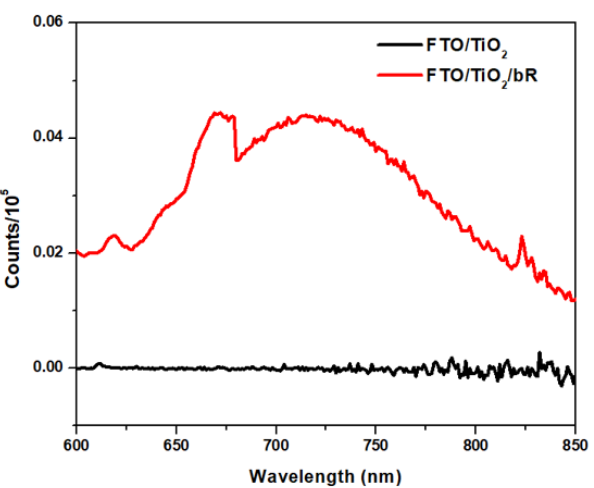

(d)

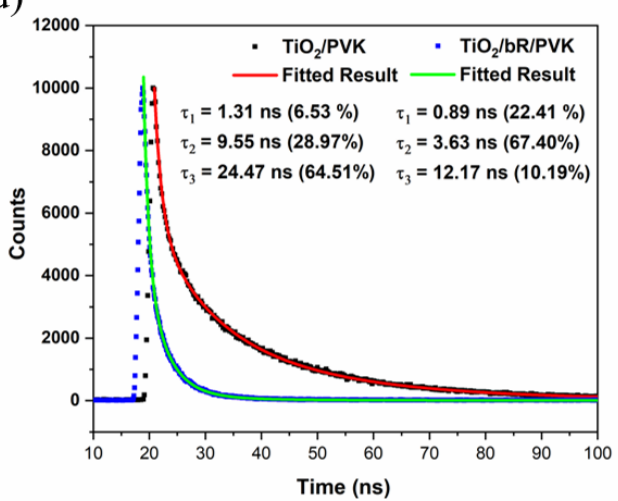

Figure 2. (a) Cross-sectional SEM of BPSC. (b) Steady-state photoluminescence (PL) spectra of $\mathrm{TiO}_{2}$ and $\mathrm{TiO}_{2} /$ bR layers. (c) Steady-state and (d) time-resolved PL spectra $\mathrm{TiO}_{2} /$ perovskite layer and $\mathrm{TiO}_{2} / \mathrm{bR} /$ perovskite layer. 
(a)

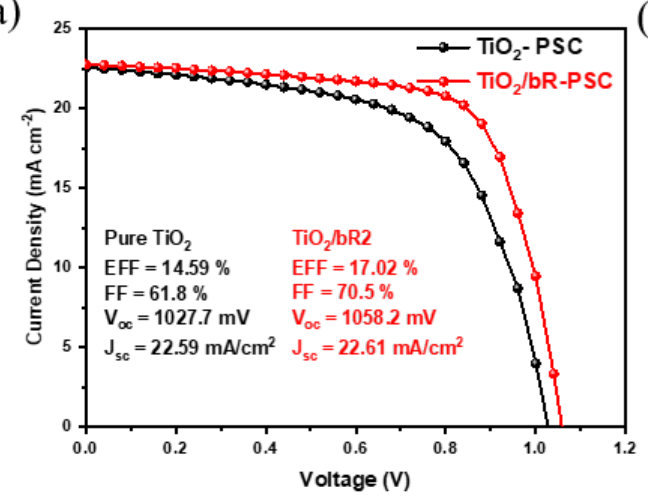

(c)

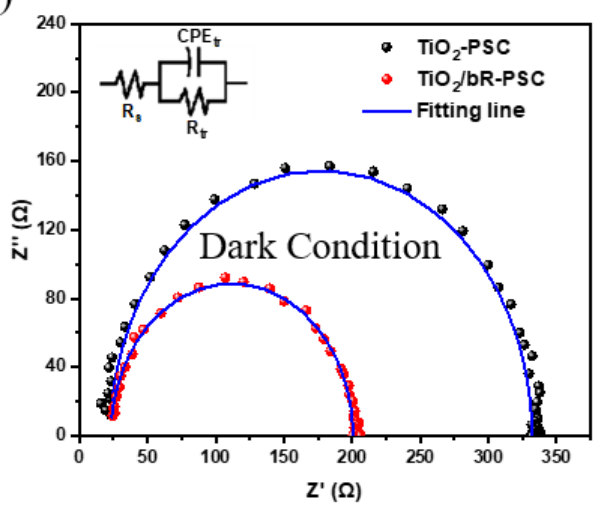

(b)

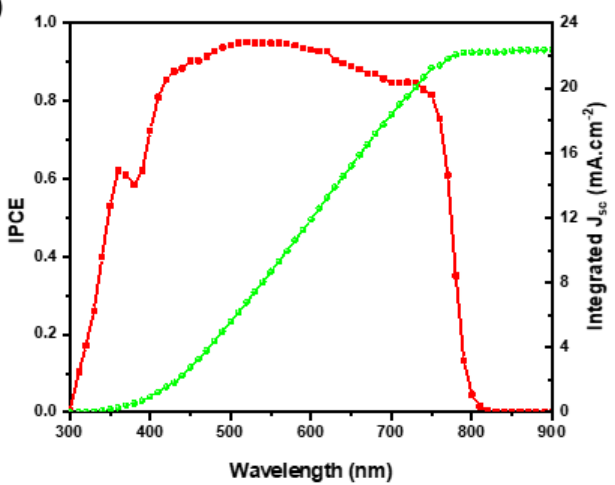

(d)

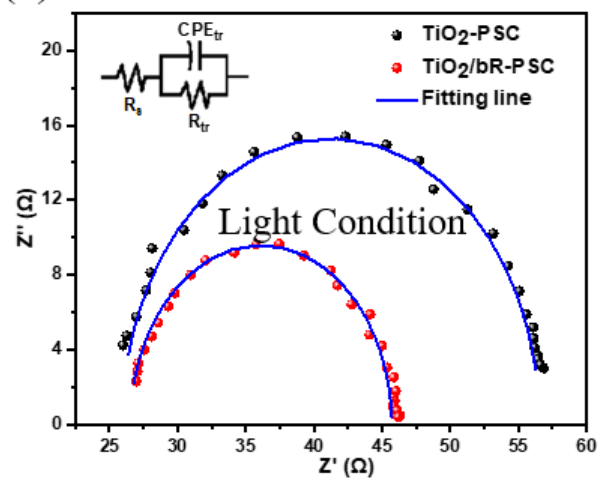

Figure 3. (a) J-V characteristics of the perovskite solar cell with and without bR integration. (b) IPCE and the integrated $\mathrm{J}_{\mathrm{sc}}$ of the BPSC. EIS of the PSCs with and without bR in the (c) dark and (d) illumination conditions. 
(a)

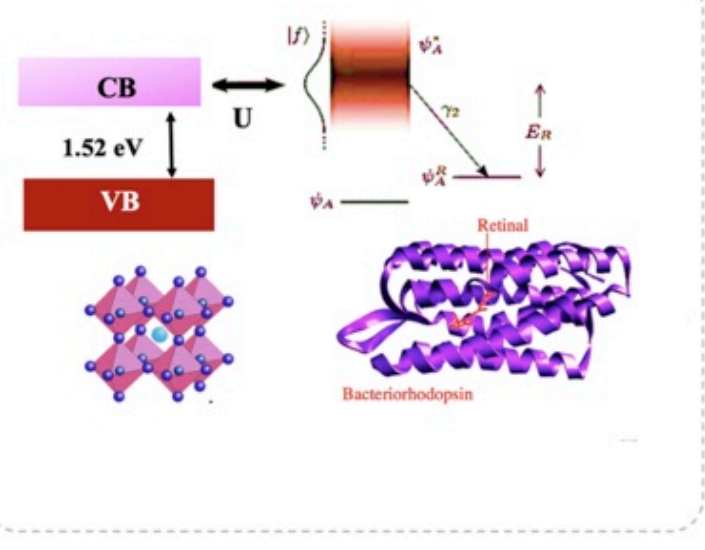

(b)

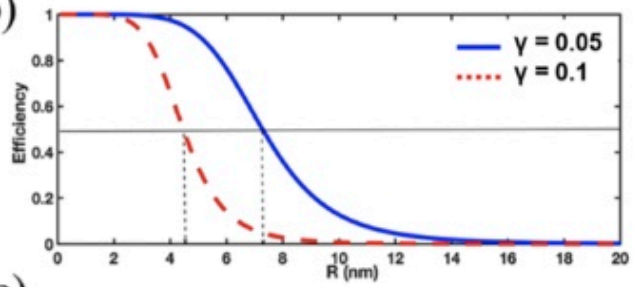

(c)

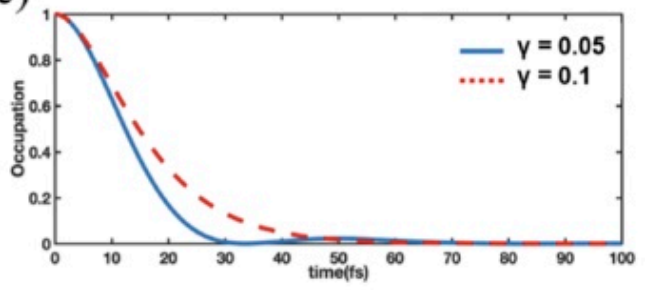

Figure 4. (a) Schematics of FRET model showing perovskite interaction with bR. (b) Exciton transport efficiency for bR-perovskite as a function of separation. (c) Occupation probability of initial excited state for $\mathrm{R}=2 \mathrm{~nm}$ as a function of time for $\gamma=0.05$ and $0.1 \mathrm{eV}$. 